\title{
CONSTRAINING PARAMETERS OF WHITE-DWARF BINARIES USING GRAVITATIONAL-WAVE AND ELECTROMAGNETIC OBSERVATIONS
}

\author{
Sweta Shah ${ }^{1,2}$ and Gijs Nelemans ${ }^{1,2,3}$ \\ ${ }^{1}$ Department of Astrophysics/IMAPP, Radboud University Nijmegen, P.O. Box 9010, 6500-GL Nijmegen, \\ The Netherlands; s.shah@astro.ru.nl \\ 2 Nikhef, National Institute for Subatomic Physics, Science Park 105, 1098-XG Amsterdam, The Netherlands \\ ${ }^{3}$ Institute for Astronomy, KU Leuven, Celestijnenlaan 200D, B-3001 Leuven, Belgium \\ Received 2013 December 20; accepted 2014 June 13; published 2014 July 17
}

\begin{abstract}
The space-based gravitational wave (GW) detector, evolved Laser Interferometer Space Antenna (eLISA) is expected to observe millions of compact Galactic binaries that populate our Milky Way. GW measurements obtained from the eLISA detector are in many cases complimentary to possible electromagnetic (EM) data. In our previous papers, we have shown that the EM data can significantly enhance our knowledge of the astrophysically relevant GW parameters of Galactic binaries, such as the amplitude and inclination. This is possible due to the presence of some strong correlations between GW parameters that are measurable by both EM and GW observations, for example, the inclination and sky position. In this paper, we quantify the constraints in the physical parameters of the white-dwarf binaries, i.e., the individual masses, chirp mass, and the distance to the source that can be obtained by combining the full set of EM measurements such as the inclination, radial velocities, distances, and/or individual masses with the $\mathrm{GW}$ measurements. We find the following $2 \sigma$ fractional uncertainties in the parameters of interest. The EM observations of distance constrain the chirp mass to 15\%-25\%, whereas EM data of a single-lined spectroscopic binary constrain the secondary mass and the distance with factors of two to $\sim 40 \%$. The single-line spectroscopic data complemented with distance constrains the secondary mass to $\sim 25 \%-30 \%$. Finally, EM data on double-lined spectroscopic binary constrain the distance to $\sim 30 \%$. All of these constraints depend on the inclination and the signal strength of the binary systems. We also find that the EM information on distance and/or the radial velocity are the most useful in improving the estimate of the secondary mass, inclination, and/or distance.
\end{abstract}

Key words: binaries: close - gravitational waves - white dwarfs

Online-only material: color figures

\section{INTRODUCTION}

Gravitational wave (GW) observations and electromagnetic (EM) observations can be used to study compact Galactic binaries independently and often these two methods provide different measurements of the same system. There are about $\sim 50$ of these binaries that have been studied in the optical, ultraviolet (UV), and X-ray wavelengths (e.g., Roelofs et al. 2010). This number is expected to grow by a factor of $\sim 100$ (Nissanke et al. 2012) when a space-based GW observatory like the evolved Laser Interferometer Space Antenna (eLISA) ${ }^{4}$ will be in operation. This detector is expected to observe millions of compact Galactic binaries with periods shorter than about a few hours (Nelemans 2009; Amaro-Seoane et al. 2013), among other astrophysical sources. Of those millions of binaries, we will be able to resolve several thousands. It has been shown (Shah et al. 2012, hereafter Paper I) that for a non-eclipsing binary system (for example AM CVn), its EM measurement of the inclination, $\iota$, can improve the error on the GW amplitude $(\mathcal{A})$ significantly depending on the strength of the GW signal and the magnitude of the EM uncertainty in the inclination. $\mathcal{A}$ is a GW parameter that is given by a combination of the masses, orbital period, and distance to the source:

$$
\mathcal{A}=\frac{4\left(G \mathcal{M}_{c}\right)^{5 / 3}}{c^{4} d}(\pi f)^{2 / 3}
$$

\footnotetext{
4 In preparation by ESA, expected launch in $\sim 2034$.
}

where $d$ is the distance to the source, $f$ is the source's GW frequency $\left(2 / P_{\text {orb }}\right)$, and $\mathcal{M}_{c}$ is the chirp mass defined as

$$
\mathcal{M}_{c} \equiv\left(m_{1} m_{2}\right)^{3 / 5} /\left(m_{1}+m_{2}\right)^{1 / 5}
$$

From the GW observations alone, one typically cannot measure the individual masses or the distance since they are degenerate via Equations (1) and (2). In the rare cases that a precise orbital decay $(\dot{f})$ can be measured from GW data, then the distance can be estimated (with the assumption that the frequency evolution is dictated by GW radiation only) by determining $\mathcal{M}_{c}$ from the measured $f$ and $\dot{f}$ via the following equation (Peters \& Mathews 1963):

$$
\dot{f}=\frac{96 \pi}{5} \frac{G^{5 / 3}}{c^{5}}\left(\pi \mathcal{M}_{c}\right)^{5 / 3} f^{11 / 3} .
$$

For the compact binaries that have been observed with the optical telescopes, a subset of which will also be detected by eLISA, their EM data often provide measurements of the orbital period $P_{\text {orb }}$, the primary mass $\left(m_{1}\right)$, sometimes the secondary mass $\left(m_{2}\right)$, the distance $(d)$, and the radial velocity amplitude $\left(K_{i}\right)$. We use these measurements for a few binaries to show the quantitative improvements in their GW and other physical parameters. Many of these binaries can/could still be found electromagnetically before or after eLISA discoveries.

We have previously shown that finding sky positions from EM data can improve the GW uncertainties on $\mathcal{A}$ and $\iota$ depending on the particular geometry and orientation of the binary systems (Shah et al. 2013). Thus we have so far quantitatively studied the improvement factors in the uncertainties of the parameters 
Table 1

GW Parameter Values of J0651

\begin{tabular}{|c|c|c|c|c|c|c|c|c|c|}
\hline & $\mathcal{A}\left(\times 10^{-22}\right)$ & $\begin{array}{c}\mathcal{M}_{c} \\
\left(M_{\odot}\right)\end{array}$ & $\begin{array}{c}\phi_{0} \\
(\mathrm{rad})\end{array}$ & $\cos \iota$ & $\begin{array}{c}f\left(\times 10^{-3}\right) \\
(\mathrm{Hz})\end{array}$ & $\begin{array}{c}\psi \\
(\mathrm{rad})\end{array}$ & $\sin \beta$ & $\begin{array}{c}\lambda \\
(\mathrm{rad})\end{array}$ & $\mathrm{S} / \mathrm{N}$ \\
\hline J0651 & $1.67^{\mathrm{a}}, 6.71^{\mathrm{b}}$ & $0.32^{\mathrm{a}}, 0.70^{\mathrm{b}}$ & $\pi$ & 0.007 & 2.61 & $\pi / 2$ & 0.101 & 1.77 & $\sim 13^{\mathrm{a}}, 50^{\mathrm{b}}$ \\
\hline
\end{tabular}

Notes.

${ }^{\text {a }}$ For $m_{1}=0.25 M_{\odot}, m_{2}=0.55 M_{\odot}$, and $d=1.0 \mathrm{kpc}$.

${ }^{\mathrm{b}}$ For $m_{1}=0.8 M_{\odot}, m_{2}=0.8 M_{\odot}$, and $d=1.0 \mathrm{kpc}$.

that can be gained from prior knowledge of parameters that are common to both GW and EM observations, for example, inclination and sky position.

In this paper, we go beyond constraining only those GW parameters that are also measured independently from the EM data. We explore various combinations of any possible EM observations and the GW measurements in constraining the useful parameters of the binaries that are astrophysically interesting, for example, the individual masses. Because their GW signal is significantly affected, we consider high-inclination (sometimes eclipsing) and (low inclination) binary systems. We review the GW data analysis methods in Section 2. In Section 3, we explore the information gained by combining EM measurements in different ways in which the EM data can be the radial velocity of one of the binary components, $K_{i}, m_{1}, m_{2}$, $d$, and $P_{\text {orb }}$. Specifically, we classify various combinations into a number of scenarios in discussing the parameter constraints.

\section{PARAMETER UNCERTAINTIES FROM GW OBSERVATIONS}

For our analyses below, we consider one of the $e L I S A$ verification binaries J065133.33+284423.3 (hereafter J0651; Brown et al. 2011). We also consider a second (hypothetical) system with higher masses, which we will refer to as "the high-mass binary." Their GW parameter values are listed in Table 1. Before looking at the EM data, we briefly recap our GW data analysis method. We have used Fisher matrix studies (e.g., Cutler 1998) to extract the GW parameter uncertainties and correlations in the GW parameters that describe the compact binary sources. Our method and application of Fisher information matrix (FIM) for $e$ LISA binaries together with their signal modeling and the noise from the detector and the Galactic foreground have been described in detail in Paper I. Most of the binaries will be monochromatic sources and such sources are completely characterized by a set of seven parameters: dimensionless amplitude $(\mathcal{A})$, frequency $(f)$, polarization angle $(\psi)$, initial GW phase $\left(\phi_{0}\right)$, inclination $(\cos \iota)$, ecliptic latitude $(\sin \beta)$, and ecliptic longitude $(\lambda)$. From the GW signal of a binary and Gaussian noise, we can use the FIM to estimate the parameter uncertainties. The inverse of the FIM is the variance-covariance matrix whose diagonal elements are the GW uncertainties and the off-diagonal elements are the correlations between the two parameters. We do the GW analyses of the above mentioned binaries for eLISA observations of two years. We note that the Fisher-based method is a quick way of computing parameter uncertainties and the correlations in which these uncertainties are estimated locally at the true parameter values, and therefore by definition the method cannot be used to sample the entire posterior distribution of the parameters. Additionally, Fisher-based results hold in the limit of strong signals with Gaussian noise (e.g., Vallisneri 2008; see also Appendix C).

The two-dimensional (2D) GW distribution in amplitude and inclination given by the variance-covariance matrix for J0651

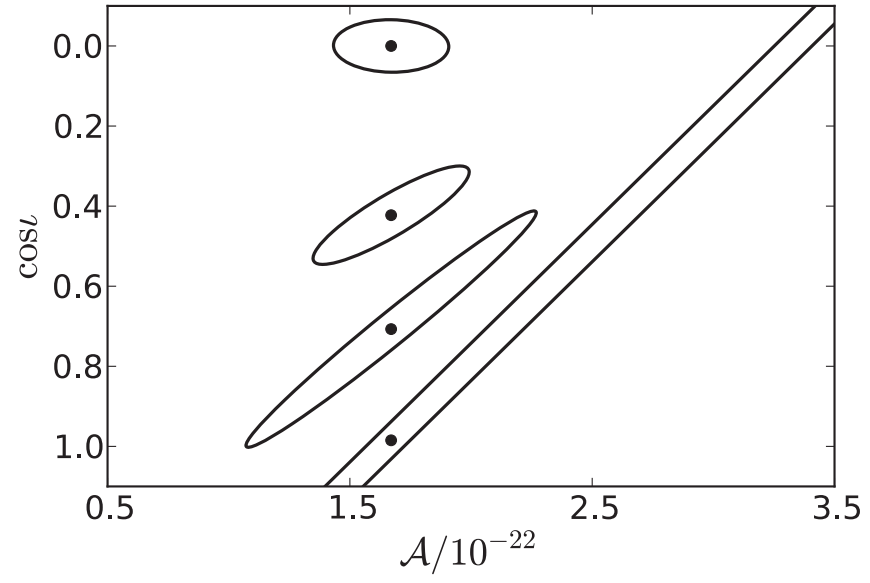

Figure 1. Two-dimensional error ellipses of $\mathcal{A}$ and $\cos \iota$ extracted from the variance-covariance matrix for the J0651 binary system with varying orientation in its $\iota$. The distributions with larger to smaller ellipses correspond to $\iota=10^{\circ}, 45^{\circ}, 65^{\circ}$, and $90^{\circ}$, respectively. The black dots are the GW parameter values (see Table 1) in which the FIM is evaluated for the corresponding orientations.

parameters are shown in Figure 1 for a number of inclinations. The largest and most highly correlated distribution is that with $\iota=10^{\circ}$ and the weakest correlation is that with $\iota=90^{\circ}$. The behavior of these distributions reflect the strength of the correlation between amplitude and inclination. As discussed in Paper I, the low-inclination systems $\iota \leqslant 45^{\circ}$ have very similar signal shapes, whereas systems with high inclinations are distinguishable by both the shape and structure for small differences in inclinations. Thus, for low-inclination binaries, a small change in $\iota$ is indistinguishable from a small change in its $\mathcal{A}$. On the other hand, for high-inclination binaries, a small change in inclination produces a noticeably different signal explaining the uncertainties in $\mathcal{A}$ and $\iota$ becoming large to small with increasing inclination. The GW uncertainties for the amplitude and inclination as a function of inclination are shown in Figure 2 for J0651 (filled circles) and the high-mass binary (open circles). The strong increase in uncertainty trends for low-inclination systems is due to the correlation between amplitude and inclination (Shah et al. 2012). Clearly, the highmass binary has a larger signal-to-noise ratio $(\mathrm{S} / \mathrm{N})$, which gives smaller uncertainties in both of its parameters shown with open circles in the figure compared to that of J0651. Observe that inclination is a cyclic parameter and is bounded between $0^{\circ} \leqslant \iota \leqslant 90^{\circ}$ and yet we get very large uncertainties from Fisher matrix for lower inclinations systems shown in the right panel of in Figure 1. This is due to the fact that Fisher matrix methods are based on the linearized signal approximation, as a result of which it is not sensitive to the bounded parameters that describe the signal model (Vallisneri 2008). In other words, in FIM, one computes the uncertainties in parameters based on variations of the signal with respect to the parameters at 

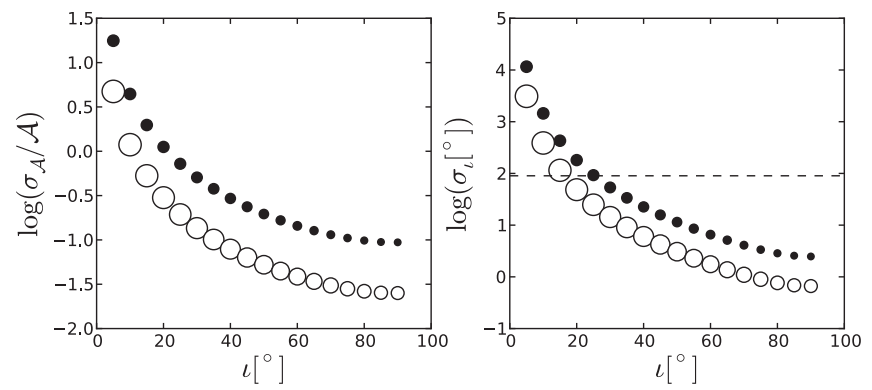

Figure 2. GW uncertainties in amplitude and inclination for J0651 as a function of inclination calculated from the Fisher matrices. Filled circles are for J0651 with $m_{1}, m_{2}=0.25,0.55 M_{\odot}$ and open circles are for the case of the high-mass binary with $m_{1}, m_{2}=0.8,0.8 M_{\odot}$. The size of the marker represents the $\mathrm{S} / \mathrm{N}$ at each inclination. The dashed line on the right panel marks the unphysical values for the inclination (see the text).

the true parameter values, and the fact that far away from the true value, the parameter has a bound is not taken into account by the FIM. When the uncertainty in a bounded parameter exceeds its physically allowed range, it means the quantity cannot be determined from GW data analysis. The dashed line in in Figure 2 indicates the value (at $90^{\circ}$ ) beyond which the uncertainties in $\iota$ imply unphysical values for the inclination. Since the low-inclination systems on the left side of the plot are affected by this, corrections have to be applied to the corresponding (overestimated) uncertainties in amplitude in the left panel by discarding the unphysical range in the inclination (Shah et al. 2013). One way to correct these unphysical values is by taking a rectangular prior on the inclination. In effect, this will cut off the posterior distribution in the parameters at the physical bounds described by the prior. Note that cutting off the error ellipses at lower inclinations in Figure 1 is reasonable because taking strict bounds far away from the real value, about which the computed Fisher uncertainties will not change the shape and slope significantly. The cut off in the posterior distributions due to rectangular priors will skew the means of the parameter distributions away from the real value (Rodriguez et al. 2013, Equation (C4)). Furthermore, we stress the fact that the Fisher matrix method is an estimate and cannot follow the posterior in detail (see Appendix C).

The normalized correlations between all seven parameters for an eclipsing and non-eclipsing orientations of J0651 are listed in the variance-covariance matrices (VCM) in Appendix A. We will make use of these parameter uncertainties and their corresponding correlations when combining with various EM data in Section 3.

\subsection{GW Information Only}

We start by considering the case where we only have the GW data. From the GW observations, the astrophysical parameters of interest for a monochromatic source are its $f, \mathcal{A}$, and $\iota$. From the GW data analysis, the frequency of the source will be very well determined, $\sigma_{f} / f \sim 10^{-6} \mathrm{~Hz}$ (e.g., Paper I) for a $10^{-3} \mathrm{~Hz}$ source, so we consider that $f$ is essentially known with a fixed value. Given that most of the binaries that we will observe with eLISA will be binary white dwarfs (WDs; Nelemans et al. 2001), we can restrict their masses to $m_{i} \in[0.1,1.4] M_{\odot}$. For simplicity, we take uniform distributions for both masses in this range. This provides a distribution in the system's chirp mass, which will provide an upper limit on the distance for the source. In Figure 3, we show these estimates in $d$ with their ninety-fifth percentile (or $2 \sigma$ uncertainties) as a function of

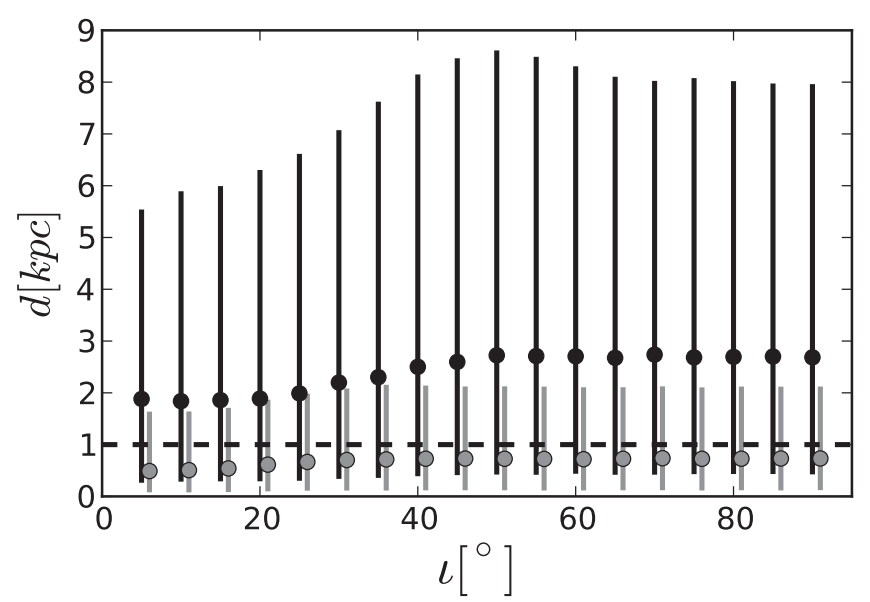

Figure 3. GW data only. The ninety-fifth percentile in distance assuming finite chirp mass for J0651 in black lines and the high-mass binary in gray lines. The black dashed line is the true value. For clarity, the constraints for the high-mass binary are shifted to the right. We do this for all the cases below.

inclination for both J0651 (black) and for the high-mass binary with equal high-mass components (gray). The black dashed line is the real value of the distance for both systems. The lower medians of distances at the lower inclinations for both systems are explained by the fact that at $\iota=5^{\circ}$, the GW distribution of $\mathcal{A}$ has a long uniform tail. This is shown in Figure 4, where we compare the distributions of $\mathcal{A}$ for two inclinations: $5^{\circ}$ (black), and $90^{\circ}$ (gray) in the left panel. For a fixed distribution of $\mathcal{M}_{c}$, the corresponding distributions in $d$ are shown in the right panel, where the solid vertical lines are the distribution medians and dashed vertical line is the real value. We can see that $\mathcal{A}_{\text {median }}^{5^{\circ}}>\mathcal{A}_{\text {median }}^{90^{\circ}}$, thus giving $d_{\text {median }}^{5^{\circ}}<d_{\text {median }}^{90^{\circ}}$ via Equation (1) for a fixed $\mathcal{M}_{c}$. Also, observe that the median distances are overestimated for J0651 for all inclinations because the real value of the median in $\mathcal{M}_{c}$ is much smaller than that computed from uniform distributions in $m_{i}$, which is the same for all inclinations. For the high-mass binary, the computed median in $\mathcal{M}_{c}$ is close to its real value, thus translating into smaller offsets in the median distances in Figure 3. In the figure, the ninetyfifth percentiles in the distance slightly increase as a function of inclination even though the uncertainties in $\mathcal{A}$ have the opposite behavior (see Figure 2). This is because the $\mathcal{M}_{c}$ has a very large fractional uncertainty compared to that of the $\mathcal{A}$ and thus the relative error uncertainties in the chirp mass dominate those in the distance, which remain roughly constant for all inclinations.

\section{COMBINING EM AND GW OBSERVATIONS}

In all the various scenarios we analyze below, we take the EM parameters with an uncertainty of $10 \%$, which is inspired by observational uncertainties of J0651. This binary is a wellknown EM source and also a guaranteed source for $e$ LISA. J0651 is an eclipsing system and such an orientation of a nearby binary allows accurate EM measurements of its orbital parameters and the masses (accuracies of $\sim 15 \%$, primary mass; $8 \%$, secondary mass) from observing the spectra, radial velocities, and eclipses of each star by the other (Brown et al. 2011). Furthermore, its rate of change of orbital period has also been measured from follow-up high speed photometry from approximately one year of data to an accuracy of $\sim 30 \%$ (Hermes et al. 2012). This will improve in the course of time. In this section, we classify specific (possible) scenarios where we could have one or more EM data on the WD binary parameters. We 

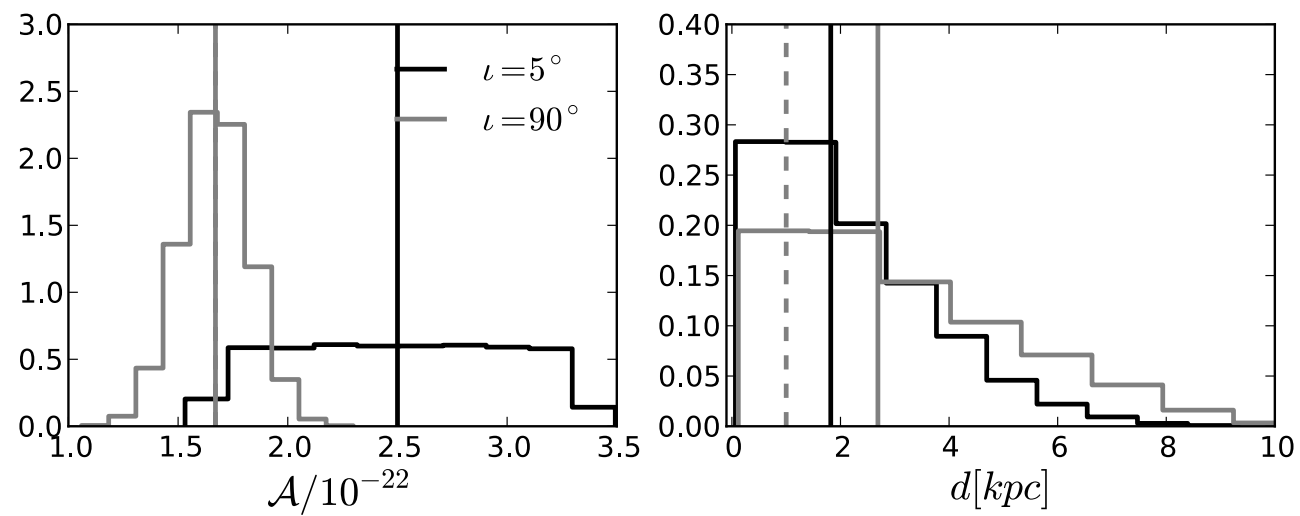

Figure 4. GW data only. Left: example of 1D distributions in $\mathcal{A}$ from GW data for two inclinations as labeled and their corresponding distributions. Right: assuming a finite $\mathcal{M}_{c}$, this gives corresponding distributions in distance. The solid lines (gray and black) are the medians of the distributions. The real values of the parameters are shown in vertical gray dashed lines. Note that in the left panel, the real value is the same as the median of the gray distribution and thus they overlap.
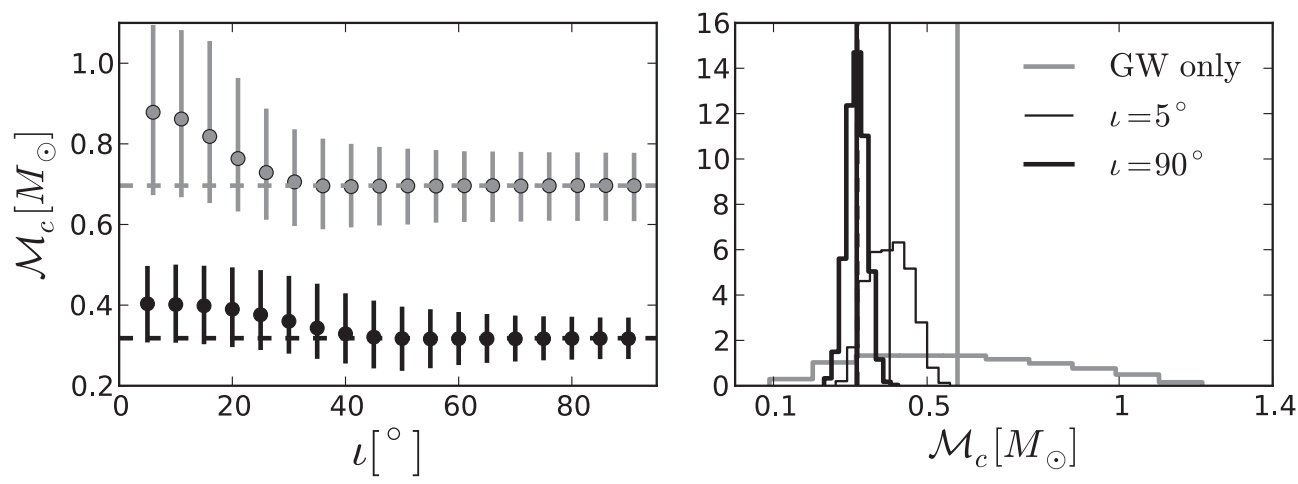

Figure 5. Scenario 1 (known distance). Left: ninety-fifth percentile in chirp mass given GW data on $\mathcal{A}$ and EM data on distance for J0651 (black) and the high-mass binary (gray). Right: example of 1D distributions in the $\mathcal{M}_{c}$ for two inclinations as labeled for J0651. The solid lines are the medians of the distributions, whereas the dashed line (overplotted on thick black solid line) is the real value of the $\mathcal{M}_{c}$. For comparison, the $\mathcal{M}_{c}$ computed from uniform distribution of masses is shown in gray.

explicitly state how much the knowledge of any of the various parameters that describe the physical properties of a binary system can be further improved if we can fold in various combinations of the existing EM and/or GW observations. $\mathrm{x}$ We construct three specific scenarios below based on the typical knowledge from the EM observations:

1. EM data on distance,

2. single-line spectroscopic data (complemented with or without the distance measurement), and

3. double-line spectroscopic data.

In all the scenarios, the GW information on amplitude, inclination, and frequency from Section 2.1 are used.

\subsection{Scenario 1: EM Observation of the Distance}

Measuring distances accurately is made feasible by the Gaia mission (de Bruijne 2012), a new astrometric satellite. Gaia is expected to measure stellar parallaxes of millions of stars with $\mu$ as accuracy, depending on how bright a star is. For example, at $1 \mathrm{kpc}$, J0651 ( $g=19.1 \mathrm{mag}$ ) would have a $\sim 300 \mu$ as accuracy in the parallax measurement corresponding to a fractional accuracy in distance of $\sim 10 \%$ (e.g., Bailer-Jones 2009). There is also some indication of the distance of the binary from its absolute magnitude. The uncertainties in $d$ from such measurements are also of the order of several percent, or $10 \%$ for the case of J0651 (Brown et al. 2011).

A sole EM measurement of the distance of a WD binary might be possible in cases where the system is identified as a WD binary but it is too faint to measure other parameters.
For instance, from the wide-field surveys, it is often possible to identify WD from their colors (Verbeek et al. 2013). Given the distance and the GW uncertainty in amplitude, we can trivially solve for the chirp mass $\mathcal{M}_{c}$ using Equation (1). The resulting probability distribution functions (pdfs) are computed by randomly drawing points from the given distributions and computing the parameter of interest for each draw. The ninetyfifth percentiles in the $\mathcal{M}_{c}$ are shown in the left panel of Figure 5 for J0651 (black) and the high-mass binary (gray) as a function of inclination. The dashed lines (black for J0651 and gray for the high-mass binary) are the real values. The decreasing medians of the chirp mass with inclination follows from the GW distributions in the amplitude that is shown in Figure 4, where the median $\mathcal{A}$ is overestimated for $\iota=5^{\circ}$ (thin black lines) compared to that of $\iota=90^{\circ}$ (thick black lines). For a fixed distribution in distance, the corresponding distribution of $\mathcal{M}_{c}$ is therefore overestimated for $\iota=5^{\circ}$ shown in the right panel of Figure 5 compared to that of $\iota=90^{\circ}$. The ninety-fifth percentiles of the chirp masses for both J0651 and the high-mass binary are affected by these overestimated medians of the amplitudes at lower inclinations, which cause significant offsets of the $\mathcal{M}_{c}$ from their respective real values as can be seen in the left panel of Figure 5. Thus at lower inclinations where the medians in the amplitude are overestimates, the ninety-fifth percentiles in the chirp mass can be interpreted as upper limits of the chirp mass. In order to calculate reliable constraints in $\mathcal{M}_{c}$ at these small inclinations, we have to do full (Bayesian) data analyses that take into account the physical priors and give us a better estimate of the expected posterior distributions in the desired 

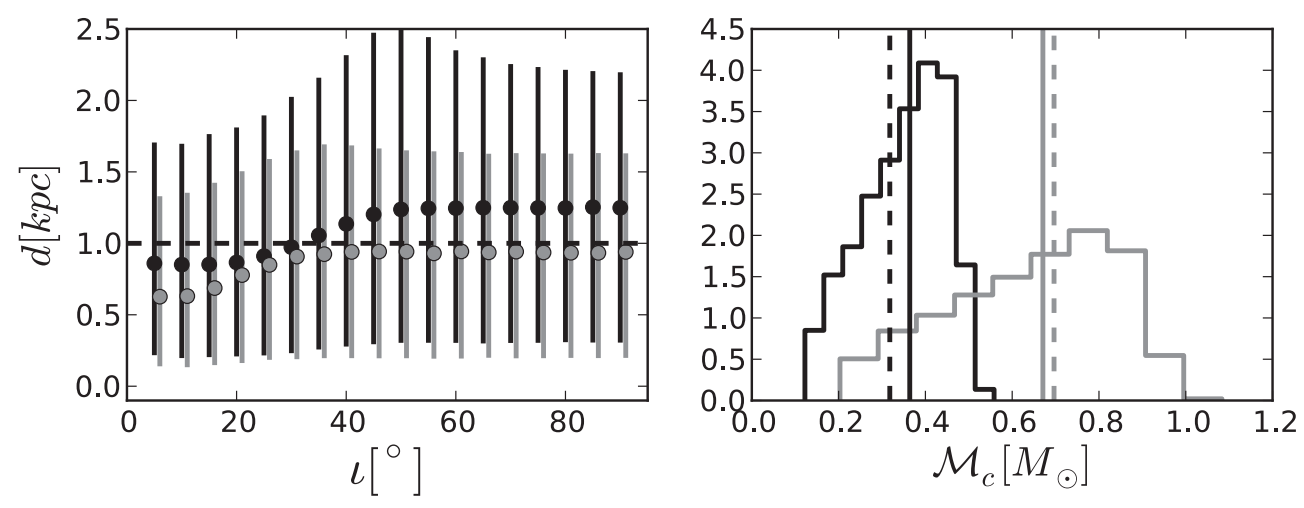

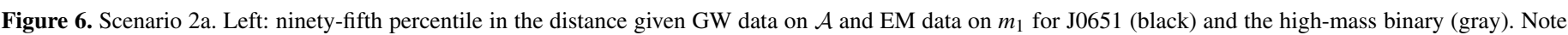

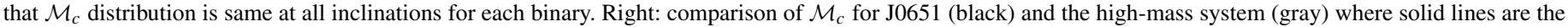
medians of the distributions and dashed lines are the real values.

parameters. The ninety-fifth percentiles in $\mathcal{M}_{c}$ for both systems decrease as a function of inclination, as is expected from the propagation of uncertainty where $\sigma_{\mathcal{M}_{c}} \propto \sigma_{\mathcal{A}}$. Thus, knowing the distance from the EM observation gives us an estimate of the chirp mass where the constraints are tighter for the higher inclination (eclipsing) systems.

\subsection{Scenario 2: EM Observations of Single-lined Spectroscopic Binary}

Some measurements are unique to EM observations, such as the radial velocity $K_{1}$, of one of the components $\left(m_{1}\right)$ of the binary:

$$
K_{1}=\sin \iota \frac{m_{2}}{\left(m_{1}+m_{2}\right)^{2 / 3}}\left(\frac{2 \pi G}{P_{\text {orb }}}\right)^{1 / 3},
$$

which can be used to measure inclination. We adopt the convention from the optical studies of the binary sources where the primary mass is the brighter object, $m_{1}$, and the dimmer secondary mass, $m_{2}$. Note that the inclination measurement from the $\mathrm{GW}$ data analysis $\iota[\mathrm{GW}]$ and from the radial velocity equation above, i.e., $\iota[\mathrm{RV}]$, are two independent measurements for the same system. We will show that these two are anticorrelated below in Section 3.2.3, yielding very useful radial velocity measurements.

\subsubsection{Scenario 2a: EM Data on $m_{1}$}

Before looking at a real single-lined spectral binary, we first consider the case that only the mass $m_{1}$ is known from the EM data. This is a viable scenario when determining $K_{1}$ is impossible and we may get an estimate of the primary mass from the photometry or the spectra. Assuming a double WD system, we take a uniform distribution for $m_{2}$, which together with the given $m_{1}$ constrains the $d$. The estimates of distance with their corresponding ninety-fifth percentiles as a function of inclination are shown in Figure 6 for both J0651 (black) and the high-mass binary (gray). The real value of distance is shown in the dashed (black) line. The offsets of the medians in the distance at low inclinations for both the binary systems can be explained in a similar way as in the previous sections, which is being due to the overestimated medians of $\mathcal{A}$ at lower inclinations as shown in the left panel of Figure 4. Additionally, the significant discrepancy between the median distance for J0651 versus the high-mass binary (at $\iota \geqslant 40^{\circ}$ ) is again due to the overestimated value of the $\mathcal{M}_{c}$ for J0651, assuming a uniform distribution $m_{2}$ distribution. This is shown in the right panel of Figure 6, where the vertical dashed lines are the corresponding true values of the $\mathcal{M}_{c}$ and the vertical solid lines are the medians of the corresponding distributions. The simulated distribution of $\mathcal{M}_{c}$ from an EM measurement of $m_{1}$ with a Gaussian width in its uncertainty together with an assumed uniform distribution in the unknown $m_{2}$ results in an overestimated median of the $\mathcal{M}_{c}$ for J0651 compared to that of the high-mass binary. This propagates overestimating the median $d$ for J0651 at higher inclinations, unlike for the high-mass binary since its median $\mathcal{M}_{c}$ is slightly underestimated. The flat priors on $m_{2}$ are affecting this and if we already know that the secondary mass is low, we may take a distribution in $m_{2}$ weighted toward lower masses, which will affect the constraints obtained in the $d$. The constraints in the distance from Figure 6 can be compared with those in Figure 3, where there was no EM information on any of the masses: the upper limits on $d$ for both J0651 and the high-mass binary are constrained by up to a factor of $\sim 4$ better when $m_{1}$ is known for both binaries with $10 \%$ accuracy.

\subsubsection{Scenario 2b: EM Data on $m_{l}$ and $K_{l}$}

In this case, we consider EM measurements of a single-lined spectroscopic binary where resolving one of the masses of the binary spectroscopically typically provides measurements on the primary mass and its radial velocity. We assume an uncertainty in radial velocity amplitude of $10 \%$ corresponding to the typical accuracy of $10 \mathrm{~km} \mathrm{~s}^{-1}$ found in the EM measurements (for, e.g., Roelofs et al. 2006). Given $m_{1}$ and $K_{1}$ from the EM data and inclination from $\mathrm{GW}$ data $\iota[\mathrm{GW}]$, we can numerically solve for $m_{2}$ via the $K_{1}$ formulation in Equation (4). Assuming it is a $\mathrm{WD}$, the $m_{2}$ is restricted to lie in [0.1-1.4] $M_{\odot}$. Then a fixed pair of $[\mathcal{A}, \iota[\mathrm{GW}]]$ and the masses give us a distance. We calculate the resulting distributions in $m_{2}$ and the distance from the Gaussian distributions of $m_{1}$ and $K_{1}$ about their typical EM uncertainties and GW distributions in the inclination and amplitude. The ninety-fifth percentile in the secondary mass and the distance are shown in Figure 7 as a function of inclination for both J0651 (black) and the high-mass binary (gray). Like in the scenarios discussed above, for the lowest inclinations, the overestimated FIM uncertainties of $\mathcal{A}$ propagate into erroneous constraints on $m_{2}$ and $d$. Thus at lower inclinations, we have to use Bayesian methods to get accurate GW uncertainties. Observe that the ninety-fifth percentile in the $m_{2}$ and the distance is roughly similar and large from $5^{\circ}<\iota<45^{\circ}$. This is again due to the influence of the GW distributions in $\mathcal{A}$ at the lower inclinations, which have uniform distributions resulting into overestimated 

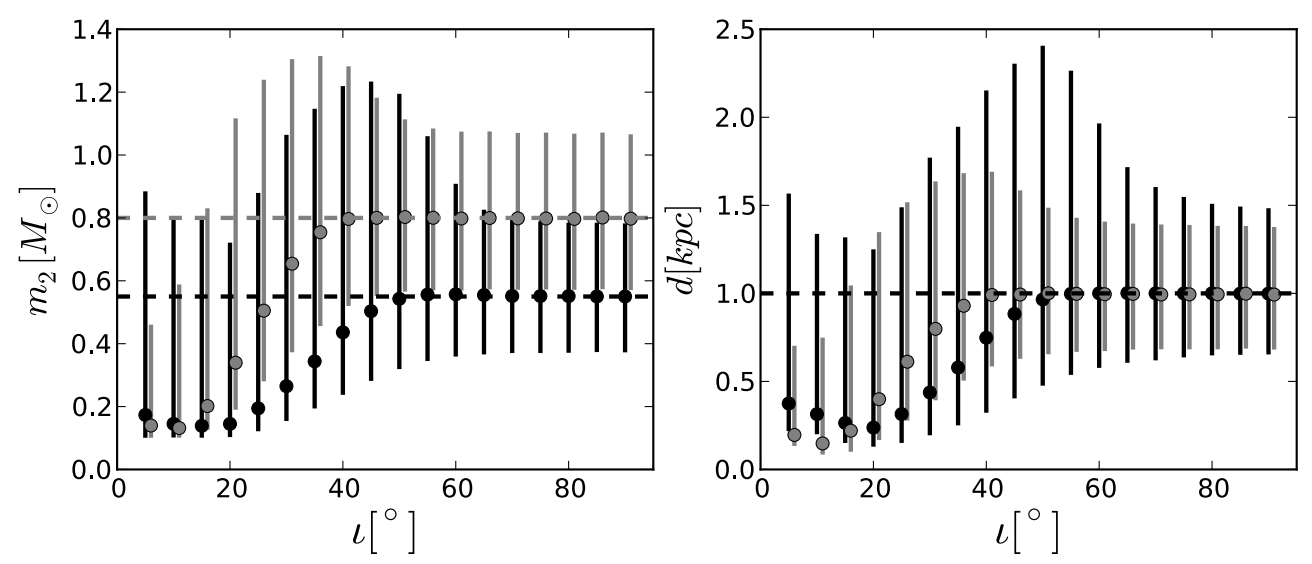

Figure 7. Scenario 2b. Constraints on the secondary mass and distance from combining single spectroscopic EM data: $m_{1}$ and $K_{1}$ with GW data on $\mathcal{A}$, $\iota$ for J0651 (black) and the high-mass binary (gray).
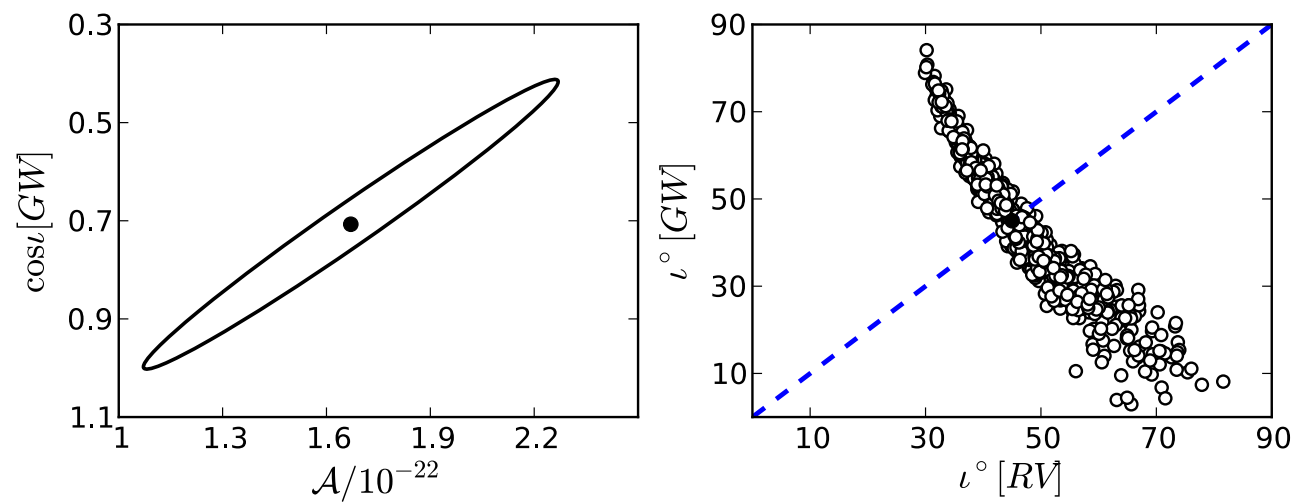

Figure 8. Relation between the inclination from GW observations vs. that from EM observations. Left: 2D error ellipse from the GW data analysis in amplitude and $\cos \iota$ for J0651 with $\iota=45^{\circ}$. Right: relation between the inclination from the left panel and the inclination from Equation (4).

(A color version of this figure is available in the online journal.)

medians (see Figure 1). However, for $\iota>45^{\circ}$, the uncertainties for both $m_{2}$ and $d$ decrease with inclination and their medians stabilize at the true values. This is caused by the fact that at higher inclinations, the medians of GW amplitudes are close to the true values of the systems where the constraints on the GW parameters are also tighter with increasing inclination. Thus, the decreasing uncertainties in $\iota[\mathrm{GW}]$ as a function of $\iota$ (see the right panel of Figure 2) should result in the same behavior of $\sigma_{m_{2}}$ via Equation (4). Since distance is computed using these $m_{2}$, the same behavior holds for the distance in the right panel.

\subsubsection{Scenario 2c: EM Data on $m_{1}, K_{l}$, and d}

Here the EM measurements of a single-lined spectroscopic binary in the previous subsection is complemented with a distance measurement from Gaia or from an estimate of the absolute magnitude. From the primary mass, $m_{1}$; distance; and amplitude, we immediately get the secondary mass, $m_{2}$. We will call this the preliminary $m_{2}$ since this can be further improved by folding in the radial velocity measurement. As mentioned before, the radial velocity measurement essentially provides an independent measurement of the inclination via Equation (4). This can be seen in the following way. The GW parameters of the non-eclipsing J0651 are $\mathcal{A}_{0}, \iota_{0}=1.67 \times 10^{-22}$, and $45^{\circ}$, whose VCM uncertainties are $\sigma_{\mathcal{A}} / \mathcal{A}=0.231$ and $\sigma_{\iota}=$ $0.75 \mathrm{rad}$, respectively. We also take a fixed radial velocity, $K_{0}$ corresponding to $m_{1}, m_{2}$ (listed in Table 1), and $\iota_{0}$. The 2D Gaussian distribution from GW data with $1 \sigma$ uncertainties for these parameters is shown in the left panel of Figure 8 .
For each randomly selected pair of $[\mathcal{A}, \cos \iota[\mathrm{GW}]]$ and for a fixed $m_{1}$ and $d$, we can solve for the $m_{2}$ from Equation (1). Using this $m_{2}$ for that fixed $m_{1}$ and $K_{0}$, we solve for $\iota[\mathrm{RV}]$. For many points randomly picked in the $[\mathcal{A}, \cos \iota[\mathrm{GW}]]$ space the computed $\iota[\mathrm{RV}]$ are compared with the corresponding $\iota[\mathrm{GW}]$ in the right panel. The inclinations measured in two ways roughly anti-correlate. However, we know that values of $\iota[\mathrm{RV}]$ that are different from $\iota[\mathrm{GW}]$ cannot be true. Thus, constraining the inclination of the system in a small area around $45^{\circ}$ along the diagonal line in the right panel also constrains $m_{2}$ and the amplitude. We make use of this in the case considered in this subsection. The preliminary $m_{2}$ and their ninety-fifth percentiles computed from EM data on $m_{1}, d$, and the GW data on $\mathcal{A}$ as a function of inclination is shown in Figure 9 as black lines for J0651 in the left panel. The same for the high-mass binary is also shown as gray lines in the right panel. From this $m_{2}$, given $m_{1}$ and $\iota$ [GW], the radial velocity, $K_{\mathrm{GW}}$ is computed and compared with the $K_{1}$ from the EM data. Since the EM measured $K_{1}$ is more precise, we keep the subset of those $K_{\mathrm{GW}}$ and the respective $\iota[\mathrm{GW}]$ weighted with a pdf of the $K_{1}$ given by

$$
\mathcal{P}_{i}=\frac{1}{\sqrt{2 \pi \sigma_{K_{1}}}} \exp \left(\frac{-0.5\left(K_{1, i}[\mathrm{GW}]-K_{1}\right)^{2}}{\sigma_{K_{1}}}\right) d K_{1} .
$$

The final reduced ninety-fifth percentiles in $m_{2}$ are shown as black lines for J0651 in left panel and for the high-mass binary in the right panel. Observe that the uncertainties in $m_{2}$ calculated in this way for lower inclinations are similar to those at higher inclinations. Thus, the advantage of folding in the $K_{1}$ 

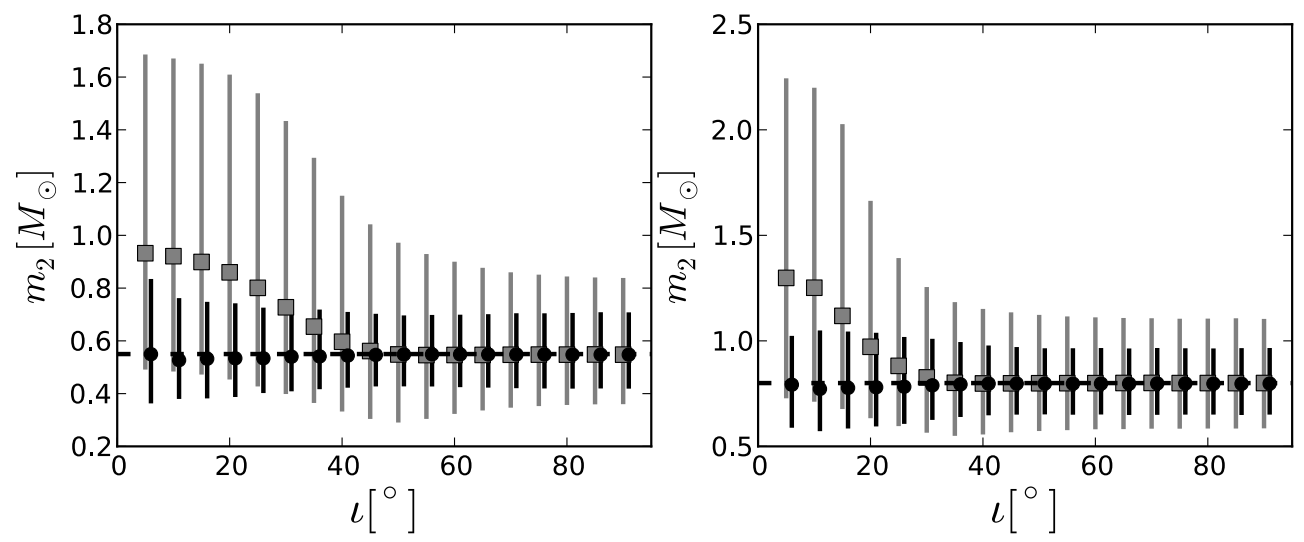

Figure 9. Scenario 2c. Same scenario as in Figure 7 with an additional EM measurement on the distance. Left: $2 \sigma$ uncertainties for the secondary mass for J0651 where the gray colored lines are constraints from EM information on $m_{1}, d$, and $\mathrm{GW} \mathcal{A}$. These reduce to the tighter constraints shown in black lines when EM data on $K_{1}$ is also used (see text). The black dashed line is the real value of $m_{2}$. Right: same for the high mass binary.
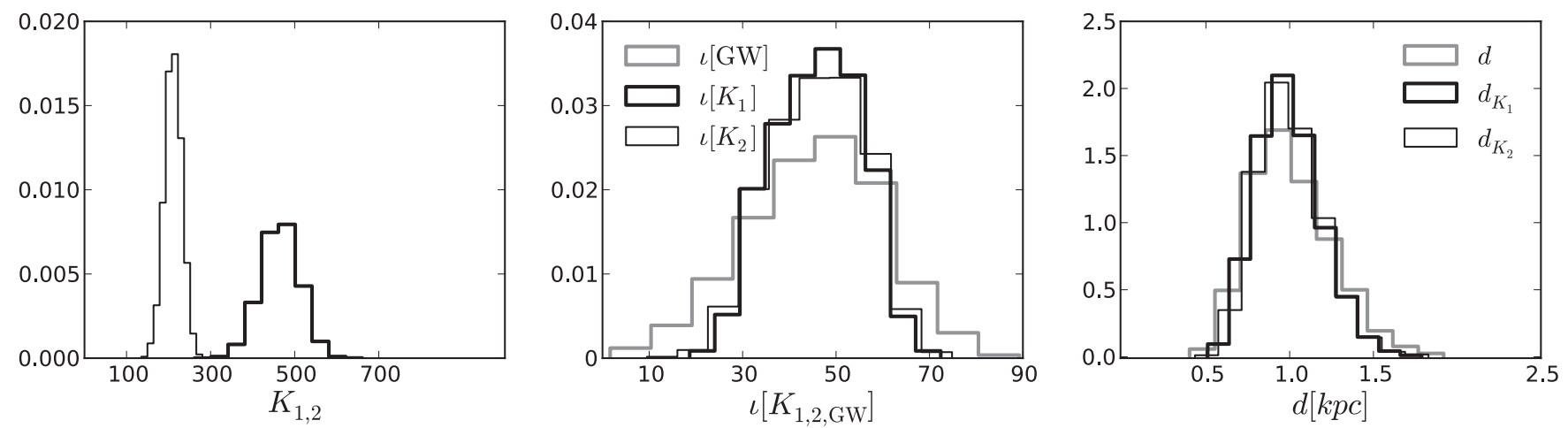

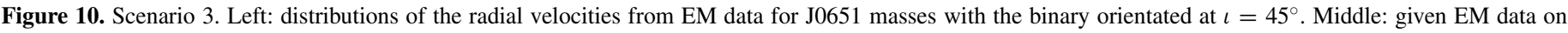

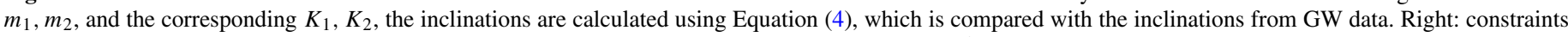
on the distance obtained solving Equation (1) with EM data on $m_{1}, K_{1}, m_{2}$, and $K_{2}$ and GW data on $\mathcal{A}$, $\iota$.

measurement is especially useful for lower-inclination useful for lower inclination systems with $\mathrm{S} / \mathrm{N} \sim 10$, where large $\mathrm{GW}$ uncertainties $\mathcal{A}$ influence the constraints of the physical parameters in question. Furthermore, the constraints in $m_{2}$ can also be compared with the previous case in Figure 7, where we find that for the single-lined spectroscopic binary, knowing its distance to $10 \%$ significantly improves knowledge of the secondary mass at lower inclinations.

The key point in Scenarios $2 \mathrm{~b}$ and $2 \mathrm{c}$ is that not all the $[\mathcal{A}, \iota[\mathrm{GW}]]$ pairs are consistent with the EM observations. Therefore both constraints on the GW data and other parameters also constrain the GW error ellipses. The $2 \sigma$ uncertainties in the $\mathrm{GW}$ amplitude and GW inclination for these scenarios are shown in the Appendix B.

\subsection{Scenario 3: EM Data on $m_{1}, K_{1}, m_{2}$, and $K_{2}$}

In this section, we consider EM observations of a double-lined spectroscopic binary, which translates to a set of measurements in the mass and radial velocities for each of the components: $m_{1}$ and $K_{1}$, and $m_{2}$ and $K_{2}$. Given the two masses and GW measurement on the amplitude, we can immediately compute a preliminary distance. Additionally, we can also derive two sets of inclinations independently from the individual radial velocities and the masses, $\iota_{\mathrm{K}_{1}}$, and $\iota_{\mathrm{K}_{2}}$ from Equation (4). These inclinations can be compared with the one measured from $\mathrm{GW}$ data, $\iota[\mathrm{GW}]$. At lower inclinations, large uncertainties in $\iota[\mathrm{GW}]$ essentially imply that those systems' inclinations are undetermined and this also affects the amplitude due to the strong correlation between them. Thus, the independent estimates of $\iota_{\mathrm{K}_{1}}$ and $\iota_{\mathrm{K}_{2}}$ from the EM data can be useful in constraining the GW amplitude. This reduced amplitude will further constrain the distance which is shown in the third panel in Figure 10. In the figure, both the observed $K_{1}$, and $K_{2}$ are shown in the left panel with thick and thin black lines, respectively. The inclination and the distance given the GW amplitude and both the masses are shown with gray lines in the middle and right panels, respectively. Both the inclination and distance derived from $K_{1}$ and $K_{2}$ are plotted with thick and thin black lines, respectively. Observe that a $10 \%$ fractional error in each $K_{1}$ and $K_{2}$ translate into similar uncertainties of the distance and thus in the following figures, we show the constraints that use only the $K_{1}$ data. The constrained distances estimated in this way as a function of inclination are shown in Figure 11 for J0651 in the left panel (black) and the high-mass binary in the right panel (also black). The gray lines in both panels are the ninety-fifth percentiles in $d$ using only the masses from the EM data and the GW amplitude. Observe that at lower inclinations, knowing masses and a radial velocity can improve the constraint in distances significantly. The uncertainties are smaller for J0651 at the lowest inclinations because the relative $10 \%$ uncertainties in $K_{1}$ have lower absolute uncertainties that propagate into the uncertainties of the distance.

Note that in practice, EM data typically provide measurements of both the masses and only one of the radial velocities with $\sim 10 \%$ precision. From the radial velocity formulation, we have the relation $m_{1} / m_{2}=K_{2} / K_{1}$, which can be used to compute the remaining $K_{i}$. This provides a consistency check between EM and GW data. The EM data can be used to derive 

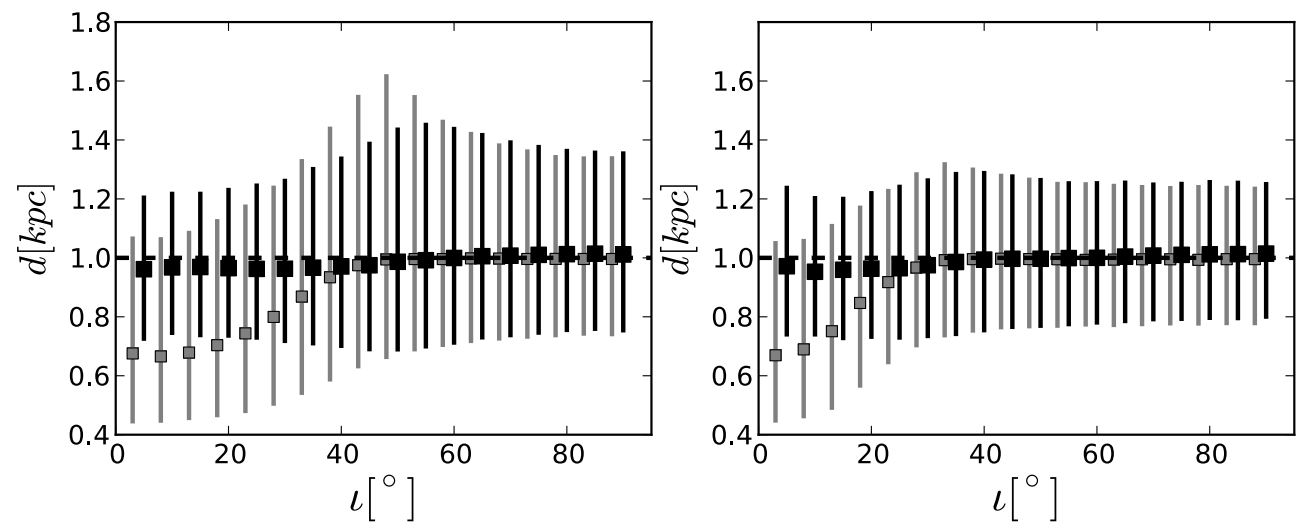

Figure 11. Scenario 3. Same as in Figure 10, but for all inclinations for J0651 in the left panel and the high-mass binary in right panel. The constraints in black lines use $\iota\left[K_{1}\right]$ and the constraints in gray use $\iota[\mathrm{GW}]$. The dashed black line shows the real values of $d$.
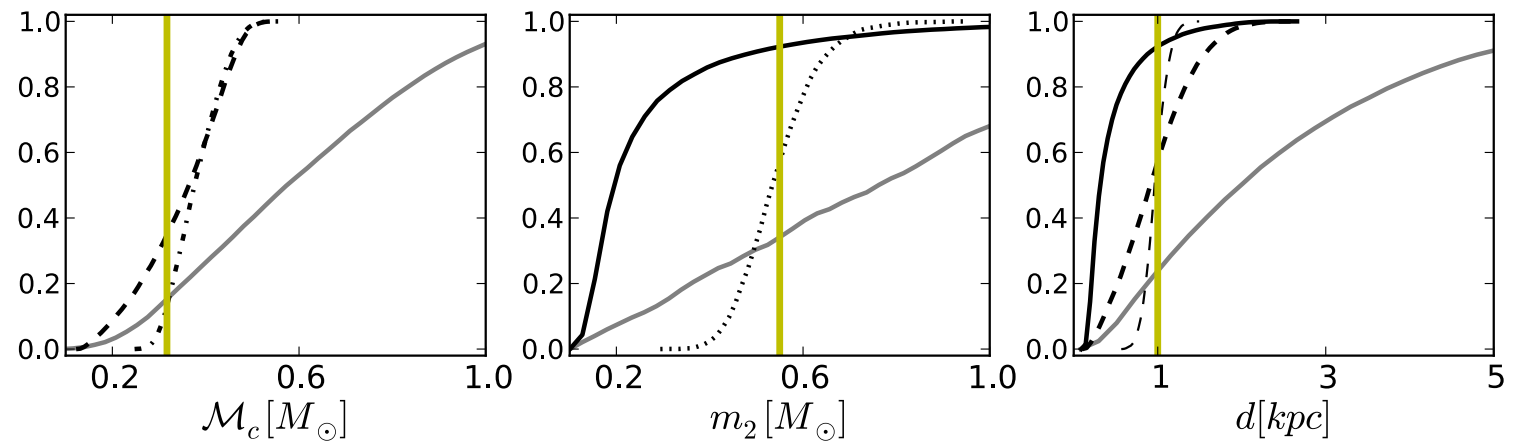

Figure 12. Comparison of normalized CDFs in $\mathcal{M}_{c}, m_{2}$, and $d$ for all the scenarios above for J0651 with $\iota=25^{\circ}$. The vertical lines (yellow) in all the panels are the true values of the parameters. The solid gray curves are CDFs for the parameters when only GW data is available. Curves in dash-dotted lines are constraints for Scenario 1 (known distance $d$ ), dashed curves are for Scenario 2a (known primary mass $m_{1}$ ), solid curves are for Scenario $2 \mathrm{~b}$ (known primary mass $m_{1}$ and radial velocity $K_{1}$ ), dotted curves are for Scenario $2 \mathrm{c}$ (known $m_{1}, K_{1}$, and $d$ ), and thin dashed lines are for Scenario 3 (known both masses $m_{1}, m_{1}$ and radial velocities $\left.K_{1}, K_{2}\right)$.

(A color version of this figure is available in the online journal.)

the inclination measured from the radial velocities, $\iota[R V]$, which can be verified against the $\iota[\mathrm{GW}]$ as shown in the middle panel of Figure 10.

\section{CONCLUSIONS}

We have quantified the possible constraints/improvements in the physical parameters of the WD binaries that are observable by the eLISA detector in the future when combined with the EM data. We do this for the binary parameters that are astrophysically interesting (masses and distance). For the GW observations from eLISA, we calculate the source's variance-covariance matrix using the Fisher methods where the Galactic binary source is described by seven parameters (eight, if $\dot{f}$ is measurable). We have taken J0651 and a higher-mass binary in our analysis where J0651 is a verification source for eLISA. We consider various possible cases depending on the availability of the EM measurements and combine those with GW uncertainties in the amplitude and inclination in order to solve for the unknown parameters as a function of inclinations for both J0651 and the high-mass binary. For clarity, we list all the cases below.

1. GW data only. Assuming a double WD system, this scenario somewhat constrains the distance.

2. Scenario 1. GW data + distance $d$ : This scenario constrains the chirp mass $\mathcal{M}_{c}$.

3. Scenario $2 a$. GW data + primary mass $m_{1}$ : This scenario constrains the chirp mass and the distance.
4. Scenario $2 b$. GW data + single-lined spectroscopic binary, i.e., $m_{1}$ and $K_{1}$ : This scenario constrains the secondary mass $m_{2}$ and the distance.

5. Scenario $2 c$. GW data + single-lined spectroscopic binary $+d$ : This scenario also constrains the secondary mass $m_{2}$.

6. Scenario 3. GW data + double-lined spectroscopic binary, i.e., $m_{1}$ and $K_{1}$, and $m_{2}$ and $K_{2}$. This scenario constrains the distance.

All $1 \sigma$ EM accuracies are taken to be $10 \%$ of the real/ measured values, which is inspired by several EM observations. We compare below the constraints in the physical parameters of interest: secondary mass $m_{2}$, chirp mass $\mathcal{M}_{c}$, and the distance $d$ as a function of the scenarios depending on the EM information available. Since the GW parameter uncertainties are significantly different for a low inclination (face-on) orientation than for a high inclination (edge-on) orientation, we do the comparison for a non-eclipsing J0651 with $\iota=25^{\circ}$ and an almost eclipsing J0651 with $\iota=85^{\circ}$ in Figures 12 and 13, respectively, and conclude the following.

1. Constraints on chirp mass, $\mathcal{M}_{c}$. In the left panels of Figures 12 and 13, EM data on $d$ constrain the ninety-fifth percentile of the system's chirp mass (dash-dotted line) to $0.38_{-0.09}^{+0.11} M_{\odot}$ and $0.32_{-0.05}^{+0.05} M_{\odot}$ for face-on and eclipsing J0651, respectively. EM data on $m_{1}$ constrain the $\mathcal{M}_{c}$ (thick dashed line) to $0.36_{-0.21}^{+0.13} M_{\odot}$, which does not depend on the inclination. The normalized cumulative distributions (CDFs) of the constraints on the distance are compared to 

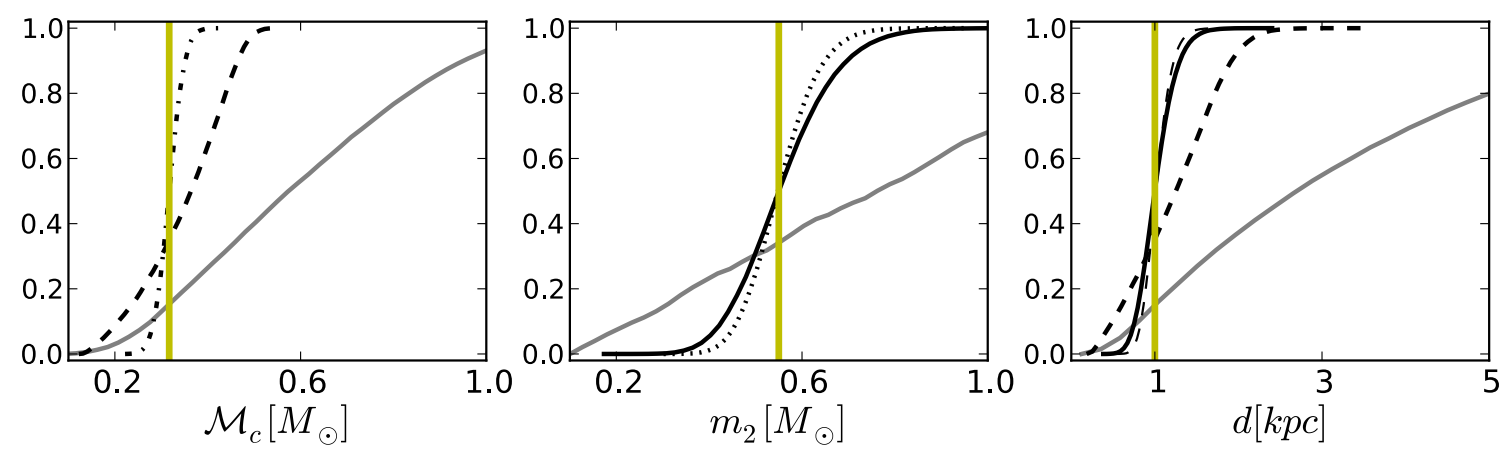

Figure 13. Same as in Figure 12, but for $\iota=85^{\circ}$.

(A color version of this figure is available in the online journal.)

those from GW data, which are shown with the gray line in both panels.

2. Constraints on secondary mass, $m_{2}$. In the middle panels of Figures 12 and 13, EM data on the $m_{1}$ and $K_{1}$ constrain the ninety-fifth percentile of secondary mass, $m_{2}$ to $0.19_{-0.07}^{+0.69} M_{\odot}$ and $0.55_{-0.18}^{+0.23} M_{\odot}$ for face-on and eclipsing J0651, respectively (solid lines). The same set of data complemented with the distance further constrain the ninetyfifth percentile in $m_{2}$ with $0.55_{-0.13}^{+0.18} M_{\odot}$ and $0.55_{-0.13}^{+0.16} M_{\odot}$ for face-on and eclipsing J0651, respectively (dotted lines). For comparison, the CDFs of $m_{2}$ using only the GW data are shown in gray.

3. Constraints on distance, $d$. In the right panels of Figures 12 and 13, EM data on $m_{1}$ constrain the distance to $0.91_{-0.69}^{+0.98}$ $\mathrm{kpc}$ and $1.25_{-0.95}^{+0.95} \mathrm{kpc}$ for face-on and eclipsing J0651, respectively (thick dashed lines). EM data on the $m_{1}$ and $K_{1}$ constrain the ninety-fifth percentile in $d$ with $0.32_{-0.16}^{+1.17} \mathrm{kpc}$ and with $0.99_{-0.35}^{+0.49} \mathrm{kpc}$ accuracy for face-on and eclipsing J0651, respectively (solid lines). EM data on $m_{1}, m_{2}, K_{1}$, and $K_{2}$ constrain the ninety-fifth percentile in $d$ to $0.96_{-0.24}^{+0.29}$ $\mathrm{kpc}$ and $1.01_{-0.26}^{+0.35} \mathrm{kpc}$ for face-on and eclipsing J0651, respectively (thin dashed line). For comparison, the CDFs of $d$ using only the GW data and the assumption that the masses are WDs are shown in gray.

Thus, knowing distance and/or radial velocity of the primary component can significantly improve our knowledge of the binary system. These constraints change as a function of inclination of the binary as shown in previous sections. In a forthcoming paper, we will address the effect on these improvements by including the (possible) EM measurements of rate of change of the orbital period.

This work was supported by funding from FOM. We are very grateful to Michele Vallisneri for providing support with the Synthetic LISA and Lisasolve software.

\section{APPENDIX A}

\section{VARIANCE-COVARIANCE MATRIXES OF J0651}

We have listed the VCM matrices for the J0651 system with eclipsing and non-eclipsing configurations in our analysis. There are seven parameters that describe them, which are listen in the first row of the matrices below. For each binary, the values are listed in the row with $\theta_{i}$. The diagonal elements are the absolute uncertainties in each the seven parameters and the off-diagonal elements are the normalized correlations, i.e., $\mathrm{c}_{i i}=\sqrt{\mathcal{C}_{i i}} \equiv \sigma_{i}, \mathrm{c}_{i j}=\left(\mathcal{C}_{i j} / \sqrt{\mathcal{C}_{i i} \mathcal{C}_{j j}}\right)$. The strong correlations between parameters (i.e., whose magnitudes are $\geqslant 0.9$ ) are marked in bold in the VCMs below. These correlations have been explained in Paper I.

VCM 1. Eclipsing J0651 $\left(\iota=5^{\circ}\right), \mathrm{S} / \mathrm{N}=10.5$.

$\left.\begin{array}{l|ccccccc} & \mathcal{A} & \phi_{0} & \cos \iota & f & \psi & \sin \beta & \multicolumn{1}{c}{\lambda} \\ \theta_{i} & \left(1.67 \times 10^{-22}\right. & \pi & 0.007 & 2.61 \times 10^{-3} & \pi / 2 & -0.08 & \multicolumn{1}{c}{2.10} \\ \hline \mathcal{A} & 0.08 \times 10^{-23} & -0.0 & 0.0 & 0.01 & 0.02 & 0.03 & -0.06 \\ \phi_{0} & & 0.907 & -0.01 & -\mathbf{0 . 9 1} & 0.01 & 0.11 & 0.08 \\ \cos \iota & & & 0.172 & 0.01 & -0.01 & 0.07 & -0.33 \\ f & & & & 2.982 \times 10^{-10} & -0.01 & -0.08 & -0.15 \\ \psi & & & & & 0.035 & -0.02 & 0.05 \\ \sin \beta & & & & & & 0.059 & 0.08 \\ \lambda & & & & & & & 0.017\end{array}\right)$

VCM 2. Non-eclipsing J0651 $\left(\iota=45^{\circ}\right), \mathrm{S} / \mathrm{N}=24$.

$\left.\begin{array}{l|ccccccc}\multicolumn{1}{c}{\mathcal{A}} & \phi_{0} & \cos \iota & f & \psi & \sin \beta & \multicolumn{1}{c}{\lambda} \\ \theta_{i} & 1.67 \times 10^{-23} & \pi & 0.707 & 2.61 \times 10^{-3} & \pi / 2 & -0.08 & 2.10 \\ \hline \mathcal{A} & 3.86 \times 10^{-23} & 0.03 & -\mathbf{0 . 9 8} & -0.02 & 0.03 & -0.13 & 0.35 \\ \phi_{0} & & 0.739 & -0.03 & -0.19 & 0.16 & 0.15 & 0.10 \\ \cos \iota & & & 0.19 & 0.02 & -0.01 & 0.13 & -0.36 \\ f & & & & 1.688 \times 10^{-9} & -\mathbf{0 . 9 8} & -0.06 & -0.21 \\ \psi & & & & & 0.36 & 0.13 & 0.07 \\ \sin \beta & & & & & & 0.031 & -0.13 \\ \lambda & & & & & & & 0.009\end{array}\right)$

\section{APPENDIX B}

\section{CONSTRAINTS IN $\mathcal{A}$ AND 乞OF J0651}

Figure 14 shows how the error ellipses of amplitude and inclination from GW observations reduce using EM observations for the different scenarios that we described in Sections 1 and 2. Knowing one of the masses (Scenario 2a) from the EM does not constrain the $[\mathcal{A}, \iota]$ any more than the GW data alone. In other words, the $m_{2}$ and $d$ are free parameters to satisfy the amplitude. The ninety-fifth percentiles in the amplitude are shown in gray in the figure, these are the same as in the case where we have only GW data. In fact, these constraints on the amplitude decrease as a function of inclination, as expected from the GW measurements (see Figure 2). Adding an EM measurement of the measured mass's radial velocity (Scenario $2 b$ ) can constrain the $[\mathcal{A}, \iota]$ slightly or significantly depending on inclination of the system, as shown in thick black lines. Finally, complementing the mass and radial velocity of the brighter companion with the distance to the binary (Scenario 2c) significantly constrains $[\mathcal{A}, \iota]$, which is strongest for the lower inclinations as shown in the figure with thin black lines. Observe that EM information provides the strongest improvements for low-inclination systems where GW uncertainties in the amplitude and the inclination are very large. 

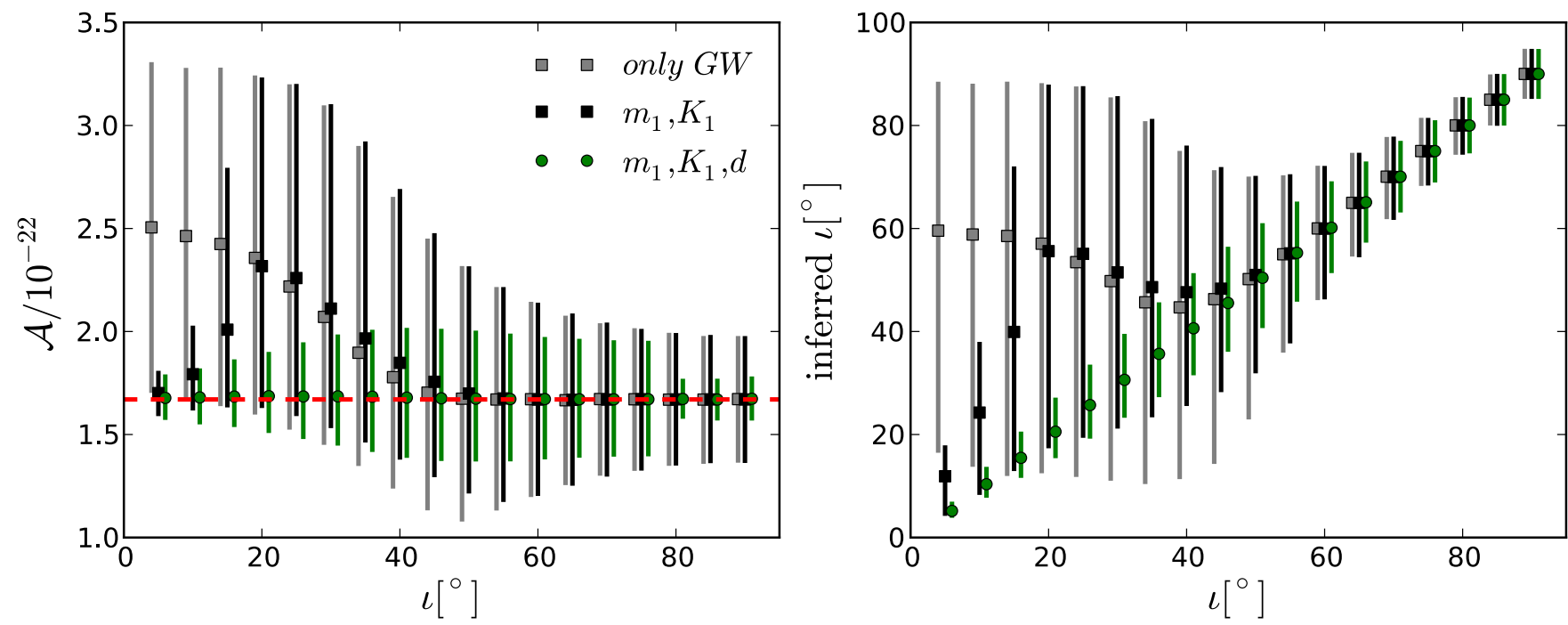

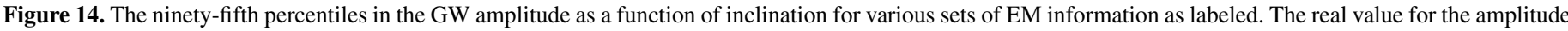
is shown by the dashed red line in the left panel.

(A color version of this figure is available in the online journal.)
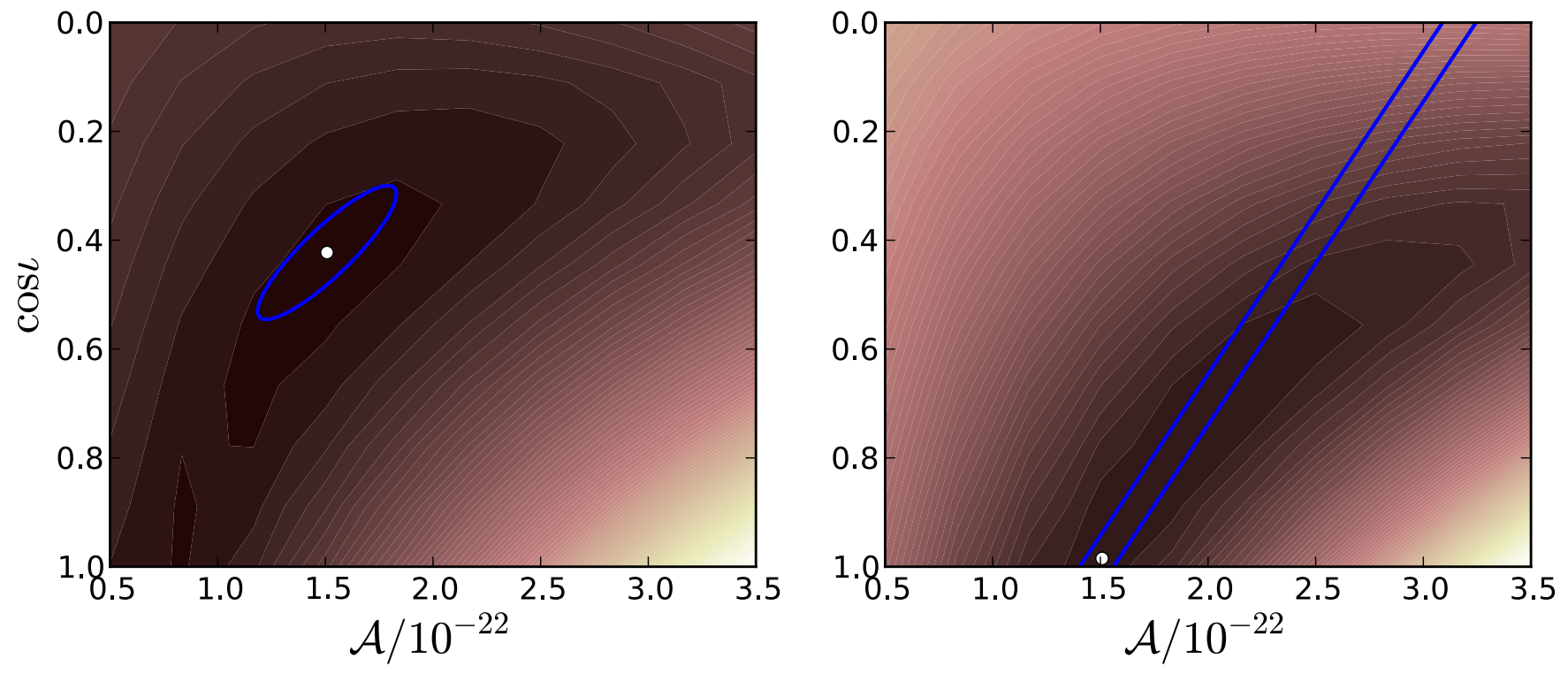

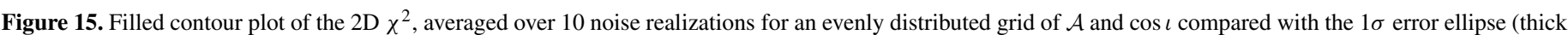

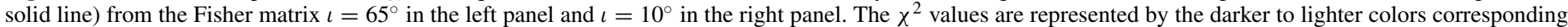
to lower to higher values, respectively.

(A color version of this figure is available in the online journal.)

\section{APPENDIX C}

\section{THE DISTRIBUTION OF $\mathcal{A}$ AND $\iota$ AT LOWER INCLINATIONS}

Here we show that while the Fisher method gives an estimate on the parameter uncertainties and correlation between them without following the posterior in detail, it also gives a reasonable estimate of the above quantities as long as the priors in the parameters are rectangular (i.e., not Gaussian) and are large enough to preserve the overall orientation of the posterior. We compute an estimate of the likelihood with a simple $\chi^{2}$ procedure on a $2 \mathrm{D}$ parameter distribution of $\mathcal{A}_{i}$ and $\cos \iota_{j}$, where the $\chi^{2}=(1 /(N-1)) \Sigma_{t=0, N}\left(h_{0}(t)-(h[i, j](t)+n(t))^{2}, h_{0}=\right.$ true signal, $h[i, j]=$ signal at a grid point and $n$ is a noise realization, $N=$ total time samples. For evenly placed parameters in a
$10 \times 10$ grid, we take the average $\chi^{2}$ computed for 10 different noise realizations. Figure 15 shows the colored contours of the 2D $\chi^{2}$ distribution for the case of $\iota=65^{\circ}$ (in the left panel) where the Fisher uncertainties are well within the physically allowed bounds. The overplotted contour (thick solid line) is the $1 \sigma$ uncertainty ellipse computed from the Fisher matrix about the true values of $\mathcal{A}$ and $\cos \iota$ labeled with the white circle. This shows that the $\chi^{2}$ distribution follows the shape and the slope of the Fisher distribution roughly, but not exactly as expected. The same is shown for $\iota=10^{\circ}$ in the right panel, where the uncertainties hit the physical bounds and both the methods show a sharp cutoff at $\cos \iota=1$. Here, we see that again the Fisher uncertainties and correlations roughly follow those of the $\chi^{2}$, but with truncations at the boundaries. The deviation in the top right is discussed in Shah et al. (2012). It was argued that 
although the results of Fisher-based uncertainties imply that the $\iota=5^{\circ}$ system is very similar to $\iota=90^{\circ}$, this is unlikely because of the anti-correlation between $\mathcal{A}$ and $\cos \iota$ at high inclinations. At $\iota \gtrsim 45^{\circ}$, correlation between $\mathcal{A}$ and $\cos \iota$ decreases $\iota$ and the high accuracy in the inclination itself actually suffices to distinguish the higher-inclination systems. Thus we expect that that $\chi^{2}$ deviates from the Fisher estimate toward the top right region of the Figure 15 in the right panel.

\section{REFERENCES}

Amaro-Seoane, P., Aoudia, S., Babak, S., et al. 2013, in GW Notes, 6 (Potsdam: Max-Planck-Institut für Gravitationsphysik), 4

Bailer-Jones, C. A. L. 2009, in IAU Symp. 254, The Galaxy Disk in Cosmological Context, ed. J. Andersen, B. m. Nordströara, \& J. Bland-Hawthorn (Cambridge: Cambridge Univ. Press), 475
Brown, W. R., Kilic, M., Hermes, J. J., et al. 2011, ApJL, 737, L23

Cutler, C. 1998, PhRvD, 57, 7089

de Bruijne, J. H. J. 2012, Ap\&SS, 341, 31

Hermes, J. J., Kilic, M., Brown, W. R., et al. 2012, ApJL, 757, L21

Nelemans, G. 2009, CQGra, 26, 094030

Nelemans, G., Yungelson, L. R., \& Portegies Zwart, S. F. 2001, A\&A, 375,890

Nissanke, S., Vallisneri, M., Nelemans, G., \& Prince, T. A. 2012, ApJ, 758,131

Peters, P. C., \& Mathews, J. 1963, PhRv, 131, 435

Rodriguez, C. L., Farr, B., Farr, W. M., \& Mandel, I. 2013, PhRvD, 88, 084013

Roelofs, G. H. A., Groot, P. J., Nelemans, G., Marsh, T. R., \& Steeghs, D. 2006, MNRAS, 371, 1231

Roelofs, G. H. A., Rau, A., Marsh, T. R., et al. 2010, ApJL, 711, L138

Shah, S., Nelemans, G., \& van der Sluys, M. 2013, A\&A, 553, A82

Shah, S., van der Sluys, M., \& Nelemans, G. 2012, A\&A, 544, A153

Vallisneri, M. 2008, PhRvD, 77, 042001

Verbeek, K., Groot, P. J., Nelemans, G., et al. 2013, MNRAS, 434, 2727 